\title{
Role of polyomavirus in emerging secondary hypofunction of marrow graft following allogeneic bone marrow transplantation in adults
}

Tatiana A. Rudakova, Yuriy A. Eismont, Ivan S. Moiseev, Ludmila S. Zubarovskaya, Alexander D. Kulagin, Boris V. Afanasyev

R.M. Gorbacheva Memorial Research Institute of Children Oncology, Hematology and Transplantation, The First St. Petersburg State I. Pavlov Medical University, St. Petersburg, Russian Federation

Contact: Dr. Tatiana A. Rudakova

E-mail: t_a_rudakova@mail.ru

\section{Background}

Poor function of haematopoietic transplant is considered an important complication following allogeneic bone marrow transplantation (allo-HSCT). According to different reports, incidence of BK polyomavirus (BKV) reactivation following allo-HSCT can be as high as 33-54\% (1). Relation between $\mathrm{BK}$ viruria (or viremia) and the risk of hemorrhagic cystitis is well studied in HSCT patients (1-3). Cases of BKV-associated encephalitis and pneumonitis have been also reported (2). Some studies suggest correlation between BK virus reactivation and cytopenia in both renal (1) and bone marrow transplant recipients (2-4). Taking into account susceptibility of different cell types to BKV infection, including peripheral leukocytes (4), one may suggest a clinical form of BKV disease manifesting as bone marrow transplant insufficiency.

\section{Objective}

The aim of our study was to assess a role of BKV infection in the development of secondary graft hypofunction following allogeneic HSCT.

\section{Materials and methods}

We carried out a retrospective analysis of 328 patients with different oncohematological disorders (18 to 77 y.o., a median of 31 years), who underwent allogeneic HSCT (matched related donors, 89; matched unrelated donors, 211; haploidentical donors, 28) during the period from 2014 to 2016. Myeloablative conditioning regimen was applied in 75 patients (23\% of total), reduced-intensity regimens were used in 253 cases (77\% of total). Characteristics of the group are shown in Table 1.

Table 1. Clinical characteristics of the group under study ( $n=328$ )

\begin{tabular}{|l|l|l|l|}
\hline Type of donors & MUD & MRD & Haplo \\
\hline Number of cases & 211 & 89 & 28 \\
\hline Observation terms, days (median+range) & $147(6-729)$ & $207(2-802)$ & $46(8-599)$ \\
\hline Active malignant process at HSCT, \% of total & $63(30 \%)$ & $28(31 \%)$ & $11(39 \%)$ \\
\hline Age at transplant, years (median+range) & $33(18-70)$ & $31(18-59)$ & $25(18-58)$ \\
\hline Gender, females & $99(47 \%)$ & $38(43 \%)$ & $10(35 \%)$ \\
\hline $\begin{array}{l}\text { Primary diagnosis: } \\
\text { AML }\end{array}$ & $96(45 \%)$ & $34(38 \%)$ & $15(54 \%)$ \\
\hline ALL & $49(23 \%)$ & $19(21 \%)$ & $5(18 \%)$ \\
\hline
\end{tabular}




\begin{tabular}{|l|l|l|l|}
\hline Type of donors & MUD & MRD & Haplo \\
\hline MDS & $12(5,7 \%)$ & $4(4,5 \%)$ & $1(3,5 \%)$ \\
\hline CML & $17(8 \%)$ & $10(11 \%)$ & $2(7 \%)$ \\
\hline Lymphomas & $19(9 \%)$ & $10(11 \%)$ & $3(10,5 \%)$ \\
\hline Myeloproliferative disease & $10(4,7 \%)$ & $1(1,1 \%)$ & - \\
\hline Aplastic anemia & $5(2,3 \%)$ & $11(12 \%)$ & $2(7 \%)$ \\
\hline Multiple myeloma & $3(1,4 \%)$ & - & - \\
\hline CLL & - & $3(3,4 \%)$ & - \\
\hline $\begin{array}{l}\text { Conditioning regimen } \\
\text { Myeloablative }\end{array}$ & $50(24 \%)$ & $21(24 \%)$ & $4(14 \%)$ \\
\hline Reduced-intensity & $161(76 \%)$ & $68(76 \%)$ & $24(86 \%)$ \\
\hline
\end{tabular}

Over the posttransplant period 219 patients were tested for $\mathrm{BKV}$ in different body fluids including blood plasma,urine, bone marrow aspirates, CSF etc. (Fig.1), using qualitative gene-specific PCR. BKV testing was carried out both in cases of local clinical symptoms (cystitis, encephalitis, graft failure etc.), and as a routine screening. Tests were positive in $166(78 \%)$ cases and negative in 45 patients (2\%). Altered graft functioning was evaluated as follows: primary graft failure (PGF) was identified in absence of donor hematopoiesis without clinical relapse; secondary graft hypofunction (SGH) was determined as post-engraftment cytopenia with sustained donor chimerism, or as a persisting dependence on transfusions and CSF injections with full donor chimerism and no signs of tumor relapse.

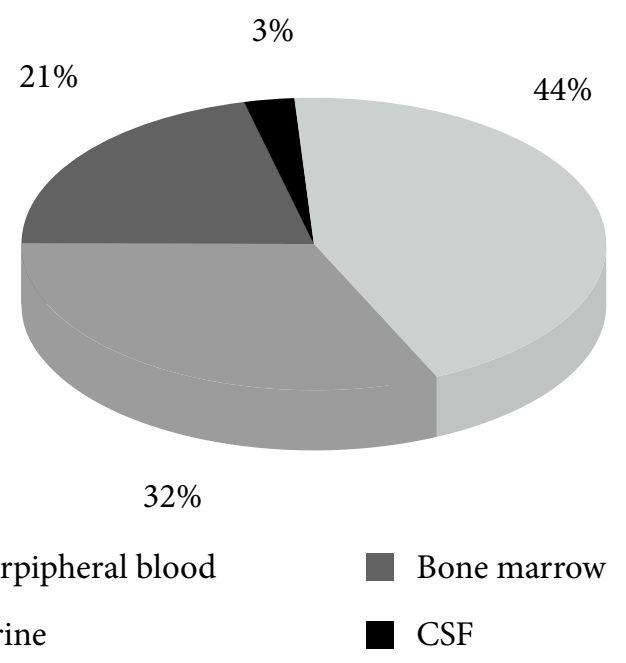

Fig. 1. Distribution of clinical samples tested for polyomavirus

\section{Results}

Poor graft function was observed in 194 patients (59\% of total); primary graft failure (PGF) was documented in 21 cases (6.4\%); secondary graft hypofunction (SGH) was revealed in 172 patients $(52.4 \%)$. BKV reactivation was found in 163 patients, including 42 cases with normally functioning graft, primary graft failure was diagnosed in 10 cases, whereas 111 patients exhibited SGH. BKV detection in bone marrow was a risk factor for $\mathrm{SGH}$, as shown by a single-factor analysis. In the patients with BKV reactivation $(n=163)$, PGF and SGH frequency was respectively $6.8 \%(n=11)$ and $68 \%(n=112)$. Among patients without BKV reactivation $(n=56)$, PGF was reported in $1.7 \%(n=1)$, and $S G H$, in $46.4 \%(n=26, p<0.002)$, as shown in Fig.2. In SGH cases, the median time for BKV detection was 46 days, thus preceding the onset of graft hypofunction (a median of 52 days).

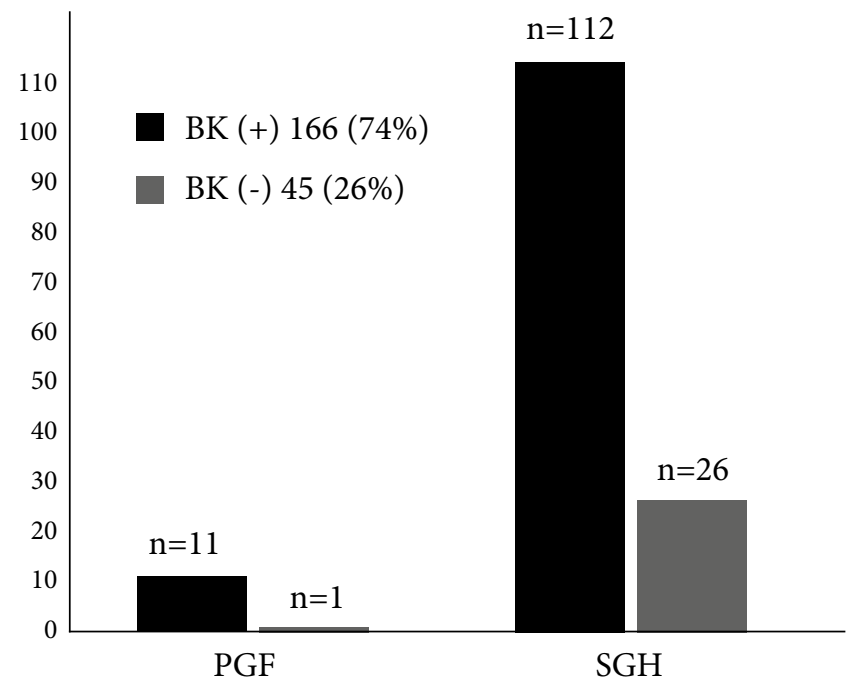

Fig. 2. Prevalence of BK virus in primary graft failure (PGF) and secondary graft hypofunction (SGH). 
BKV reactivation episodes were observed within 14 days before the onset, or during graft hy ofunction in 74 patients ( $43 \%$ of the SGH group, or $22.5 \%$ of the total HSCT cohort). Meanwhile, bone marrow samples were positive for BKV in 46 patients, i.e., $27 \%$ of all SGH cases, or $14 \%$ of total allo-HSCT cohort. However, BKV reactivation (including bone marrow affection) did not correlate with total survival after HSCT.

\section{Conclusion}

Post-transplant BKV infection may be a predisposing or triggering factor in the development of hematopoietic graft hypofunction. Further studies are required in order to obtain more detailed information about BKV replication in bone marrow and its potential effects upon post-HSCT survival.

\section{References}

1. Erard V, Storer B, Corey L, Nollkamper J, Huang M-L, Limaye A, et al. BK virus infection in hematopoietic stem cell transplant recipients: frequency, risk factors, and association with postengraftment hemorrhagic cystitis. Clin Infect Dis 2004; 39: 186

2. Reploeg MD, Storch GA, Clifford DB. BK virus: a clinical review. Clin Infect Dis 2001; 33:191-202.

3. Pambrun E, Mengelle C, Fillola G, Laharrague P, Esposito L, Cardeau-Desangles I, Del Bello A, Izopet J, Rostaing L, Kamar N. An association between BK virus replication in bone marrow and cytopenia in kidney-transplant recipients. J Transplant 2014; 2014:252914. doi: 10.1155/2014/252914.

4. Dropulic LK, Jones RJ. Polyomavirus BK infection in blood and marrow transplant recipients. Bone Marrow Transplantation 2008; 41:11-18

\section{Keywords}

Allogeneic hematopoietic stem cell transplantation, BK polyomavirus, reactivation, graft insufficiency.

\title{
Роль ВК-полиомавирусной инфекции в развитии вторичной гипофункции трансплантата костного мозга после аллогенной трансплантации костного мозга у взрослых
}

\author{
Т.А. Рудакова, Ю.А. Эйсмонт, И.С. Моисеев, Л.С. Зубаровская, А.Д. Кулагин, Б.В. Афанасьев
}

НИИ Детской онкологии, гематологии и трансплантологии им. Р.М. Горбачевой, Первый Санкт-Петербургский государственный медицинский университет им. акад. И.П. Павлова

\section{Введение}

Гипофункция трансплантата (ГТ) остаётся одной из важных проблем при проведении аллогенной трансплантации костного мозга (ТКМ). Частота реактивации ВК-полиомавируса (ВКВ) после аллогенной трансплантации костного мозга по разным данным достигает 33-54\% (1). Достаточно хорошо изучена связь между ВК-вирусурией или вирусемией и развитием геморрагического цистита у пациентов после аллоТКМ (1-3). Также имеются данные о ВК-вирусных энцефалитах и пневмонитах (2). Отдельные исследования описывают связь реактивации ВК-вируса с эпизодами цитопении у реципиентов трансплантата почки (1) и после аллоТКМ(2-4). Учитывая тропность ВК-вируса к различным типам клеток, включая лейкоциты периферической крови (4), можно предположить наличие формы ВК-болезни, проявляющейся недостаточностью трансплантата костного мозга.

\section{Цель}

Оценить значение ВК-вирусной инфекции в развитии вторичной гипофункции трансплантата после аллогенной ТКМ.

\section{Материалы и методы}

Ретроспективно были проанализированы данные 328 взрослых пациентов с различными онкогематологическими заболеваниями в возрасте от 18 до 77 лет (медиана возраста - 31 год), которым была проведена аллогенная ТГСК (родственная - в 89 случаях, неродственная - в 211 , гаплоидентичная у 28 пациентов) в период с 2014 до 2016 г. Миелоаблативный режим кондиционирования использовался у 75 (23\%) пациентов, режим со сниженной интенсивностью - в 253 случаях (77\%). Характери- 
стика пациентов приведена в Табл. 1. У 219 пациентов в посттрансплантационном периоде проводилось тестирование ВК-вирусной инфекции (BК) в различных биологических жидкостях (моча, ликвор, плазма крови, аспират костного мозга), как показано на рис.1. Диагностика полиомавирусов проводилась с помощью качественной геноспецифической ПЦР ДНК. Тестирование ВК-вируса выполнялось как при наличии клинических симптомов (цистит, энцефалит, недостаточность функции трансплантата), так и в качестве скрининга. Результаты тестирования были положительными в 166 (78\%) случаях, отрицательными в 45 (2\%) случаях. Производилась оценка функции трансплантата после ТГСК. В частности, под первичным неприживлением понималось отсутствие донорского гемопоэза без признаков основного заболевания; вторичной гипофункцией считалось возникновение цитопении после приживления и периода нормальной функции трансплантата при сохранении донорского химеризма или сохраняющаяся зависимость от трансфузий и введения КСФ при полном донорском химеризме и отсутствии признаков основного заболевания.

\section{Результаты}

Гипофукция трансплантата наблюдалась у 193 пациентов (59\%): первичное неприживление - у 21 (6,4\%), вторичная гипофункция трансплантата (ВГТ) - у 172 больных (52,4\%). Реактивация ВК-вируса была обнаружена у 163 пациентов, из них 42 пациентов имели нормальную функцию трансплантата, 10 - первичное неприживление, 111 - вторичную гипофункцию. Обнаружение ВК-вируса в костном мозге было фактором риска развития вторичной гипофункции трансплантата при однофакторном анализе. У больных с реактивацией ВК-вируса $(\mathrm{n}=163)$ частота первичного непри- живления и ВГТ составила 6,8\% $(\mathrm{n}=11)$ и $68 \%(\mathrm{n}=112)$ соответственно, тогда как у пациентов без реактивации ВК-вируса $(\mathrm{n}=56)$ первичное неприживление было документировано в $1,7 \%(\mathrm{n}=1)$, а ВГТ - в $46,4 \%$ случаев $(\mathrm{n}=26, \mathrm{p}<0,002)$. Эпизоды реактивации ВК-вируса имели место в течение 14 дней до развития или в период гипофункции трансплантата у 74 пациентов (43\% от всех пациентов с вторичной гипофункцией, 22,5\% от всех аллоТКМ). При этом в костном мозге ВК-полиомавирус обнаруживался у 46 пациентов (27\% от всех пациентов с вторичной гипофункцией, 14 \% от всех аллоТКМ). Обнаружение ВК-вируса (при медиане сроков выявления 46 сут.) предшествовало дебюту гипофункции трансплантата (медиана появления - 52 дня).

Однако в данном исследовании реактивация ВК-вируса (в том числе и в костном мозге) не коррелировала с общей выживаемостью после аллоТКМ.

\section{Выводы}

Развитие ВК-вирусной инфекции в посттрансплантационном периоде может являться предрасполагающим или пусковым фактором для формирования гипофункции трансплантата костного мозга. Дальнейшие исследования необходимы для более детального изучения возможности репликации ВК-полиомавируса в костном мозге и его влияния на выживаемость после аллоТКМ.

\section{Ключевые слова}

Аллогенная трансплантация гемопоэтических стволовых клеток, ВК-полиомавирус, реактивация, недостаточность трансплантата. 